\title{
IL-7 inhibits fibroblast TGF- $\beta$ production and signaling in pulmonary fibrosis
}

\author{
Min Huang, ${ }^{1,2}$ Sherven Sharma, ${ }^{1,2}$ Li X. Zhu, ${ }^{1}$ Michael P. Keane, ${ }^{1}$ Jie Luo, ${ }^{1}$ Ling Zhang, ${ }^{1}$ \\ Marie D. Burdick, ${ }^{1}$ Ying Q. Lin, ${ }^{1}$ Mariam Dohadwala, ${ }^{1}$ Brian Gardner, ${ }^{2}$ Raj K. Batra, ${ }^{1,2}$ \\ Robert M. Strieter, ${ }^{1}$ and Steven M. Dubinett ${ }^{1,2}$
${ }^{1}$ Department of Medicine, Division of Pulmonary and Critical Care Medicine, University of California Los Angeles School of Medicine, Los Angeles, California, USA
${ }^{2}$ Department of Medicine, Division of Pulmonary and Critical Care Medicine, Veterans Administration Greater Los Angeles \\ Healthcare System, Los Angeles, California, USA
}

Address correspondence to: Steven M. Dubinett, Division of Pulmonary and Critical Care Medicine, 37-131 CHS, 10833 Le Conte Avenue, University of California Los Angeles School of Medicine, Los Angeles, California 90095-1690, USA. Phone: (310) 794-6566; Fax: (310) 267-2829; E-mail: sdubinett@mednet.ucla.edu.

Received for publication November 20, 2001, and accepted in revised form February 21, 2002.

\begin{abstract}
Based on studies by our group and others, we hypothesized that IL-7 may possess antifibrotic activities in an IFN- $\gamma$-dependent and independent manner. Here, we have evaluated the antifibrotic therapeutic potential of IL-7 in both in vitro and in vivo pulmonary fibrosis models. IL-7 inhibited both TGF- $\beta$ production and signaling in fibroblasts and required an intact JAK1/STAT1 signal transduction pathway. IL-7-mediated inhibition of TGF- $\beta$ signaling was found to be associated with an increase in Smad7, a major inhibitory regulator in the SMAD family. In the presence of IL-7, Smad7 dominant negative fibroblasts restored TGF- $\beta$-induced collagen synthesis, indicating that an IL-7-mediated increase in Smad7 suppressed TGF- $\beta$ signaling. Consistent with these in vitro findings, recombinant IL-7 decreased bleomycin-induced pulmonary fibrosis in vivo, independent of IFN- $\gamma$. The antifibrotic activities of IL-7 merit further basic and clinical investigation for the treatment of pulmonary fibrosis.
\end{abstract}

J. Clin. Invest. 109:931-937 (2002). DOI:10.1172/JCI200214685.

\section{Introduction}

Idiopathic pulmonary fibrosis (IPF) is a devastating disease with less than a $50 \%$ five-year survival $(1,2)$. While steroids and other immunosuppressive agents serve as the standard treatment for IPF, these agents have proved inadequate (3). In most instances immunosuppressive agents do little to affect the course of the disease and have serious adverse side effects. Thus, novel therapeutic strategies are clearly needed. Recent published findings suggest novel treatment paradigms based on a more complete understanding of the pathogenesis of pulmonary fibrosis $(3,4)$.

IL-7 is a $25-\mathrm{kDa}$ glycoprotein originally isolated from bone marrow stroma cells (5). IL-7 was originally defined as a pre-B lymphocyte growth factor and was subsequently found to augment the growth of T lymphocytes (6-8). We (9) and others (10-13) have documented that IL-7 can potently enhance T cell function and IFN- $\gamma$ production. IL-7 synergizes with IL-12 in the induction of T cell proliferation, cytotoxicity, and IFN- $\gamma$ release (11). In agreement with these findings are studies indicating that IL-7 plays a role in cell-mediated immune responses characteristic of type 1 cytokines (10). We have found that IL-7 downregulates macrophage (14), fibrosarcoma, and melanoma $(15,16)$ production of TGF- $\beta$. IL-7 has the capacity to downregulate the transcriptional rate of the TGF- $\beta$ gene in murine macrophages in an IFN- $\gamma-$ independent manner (14).

TGF- $\beta$ is a critical fibrogenic factor in the development of pulmonary fibrosis. Based on the importance of TGF- $\beta$ in the pathogenesis of pulmonary fibrosis, we speculated that the most effective therapies would be those that decrease both the production and the cellular effects of TGF- $\beta$. In the current study we investigate the role of IL-7 in TGF- $\beta$ production and signaling and the potential for IL-7 as a new antifibrotic agent in the treatment of interstitial pulmonary fibrosis.

\section{Methods}

Cell culture. Pulmonary fibrosis fibroblasts (PFFs) were isolated from lung resection specimens obtained from patients with IPF. Normal fibroblasts (NFs) were isolated from patients with nonfibrotic diseases. U4A, U3A, and U4A/JAK1 cell lines were generously provided by George R. Stark (Cleveland Clinic Foundation, Cleveland, Ohio, USA). The cells were maintained in $5 \% \mathrm{CO}_{2}$ in air as monolayers at $37^{\circ} \mathrm{C}$ in $75-\mathrm{cm}^{2}$ tissue culture flasks containing $20 \mathrm{ml}$ of DMEM supplemented with $10 \%$ FBS, 100 units $/ \mathrm{ml}$ penicillin, 0.1 $\mathrm{mg} / \mathrm{ml}$ streptomycin, and $2 \mathrm{mM}$ glutamine (JRH Biosciences, Lenexa, Kansas, USA).

Cytokines and antibodies. Human activated recombinant TGF- $\beta 1\left(3.2 \times 10^{4}\right.$ units $\left./ \mu \mathrm{g}\right)$, recombinant human IL-7 


\section{Figure 1}

IL-7 decreases TGF- $\beta$ production by PFF in an IFN- $\boldsymbol{\gamma}$-independent manner. ( $\mathbf{a}$ and $\mathbf{b}$ ) Primary fibroblasts from IPF patients (PFF, $n=5$ ) or non-IPF subjects (NF, $n=5)$ were cultured in medium with or without IL-7 for 24 hours, and the culture supernatants were assayed for TGF- $\beta$ by ELISA. IL-7 decreases TGF- $\beta$ production in PFF, but not in NF. (c and $\mathbf{d}$ ) While IFN- $\gamma$ decreased TGF- $\beta$ production in both NF and PFF, IL-7 decreased TGF- $\beta$ production only in PFF. The addition of anti-IFN- $\gamma$ antibody did not alter the capacity of IL-7 to decrease TGF- $\beta$, suggesting that IL-7 acts in an IFN- $\gamma$-independent manner. ${ }^{*} P<0.01$. a

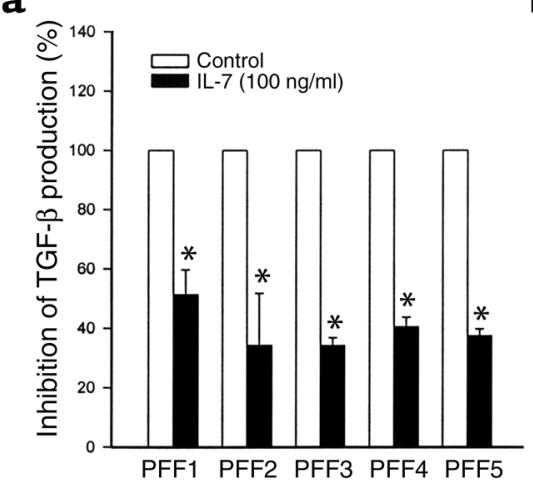

C

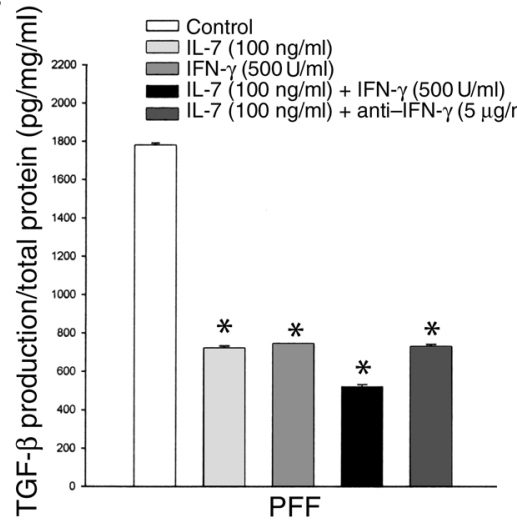

b

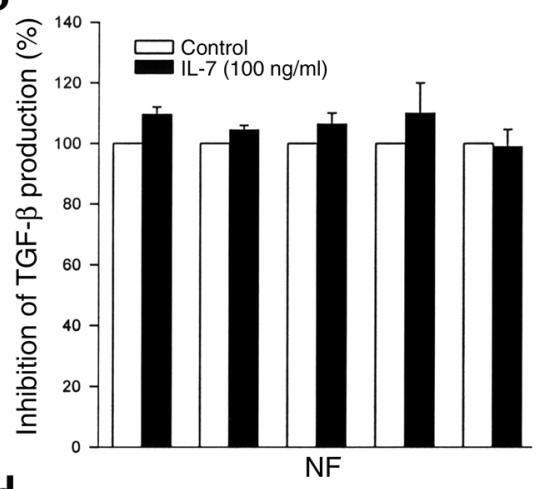

d

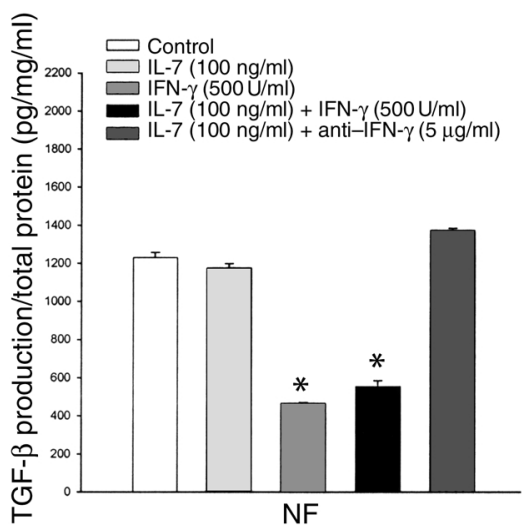

$\left(10^{5}\right.$ units $\left./ \mu \mathrm{g}\right)$, recombinant mouse IL-7 $\left(5 \times 10^{4}\right.$ units $/ \mu \mathrm{g})$, recombinant human IFN- $\gamma\left(2 \times 10^{4}\right.$ units $\left./ \mu \mathrm{g}\right)$, and mouse anti-human IFN- $\gamma$ monoclonal neutralizing antibody were obtained from R\&D Systems Inc. (Minneapolis, Minnesota, USA). Anti-mouse IFN- $\gamma$ monoclonal neutralizing antibody was purified by affinity chromatography from ascites of SCID mice, which were generated 3-4 weeks after intraperitoneal injection of $10^{6} \mathrm{R} 4-462$ hybridoma cells per mouse (American Type Culture Collection, Rockville, Maryland, USA) (17).

Dominant negative Smad7. The retroviral vector containing a full-length Smad7 cDNA was generously provided by Rik Derynk (University of San Francisco, San Francisco, California, USA). The Smad7 dominant negative mutant was constructed by a 25 -amino acid deletion at the $\mathrm{COOH}$ terminus of Smad7. After transfection to the PT-67 retroviral packaging cell line by a liposomal-mediated method (Effectene method; QIAGEN Inc., Valencia, California, USA), the supernatant containing high titer of retrovirus expressing dominant negative Smad7 was collected to transduce PFF cells.

Mice. Pathogen-free female C57BL/6 mice (6-8 weeks old) and CB17 SCID Beige mice (6-8 weeks old) were purchased from the Charles River Laboratories, Inc. (Wilmington, Massachusetts, USA) and maintained in the West Los Angeles Veterans Affairs Animal Research Facility.

Bleomycin-induced pulmonary fibrosis model. To induce pulmonary fibrosis, mice were treated with $0.15 \mathrm{U}$ bleomycin in $25 \mu 10.9 \%$ normal saline (NS) or $0.9 \% \mathrm{NS}$ alone by intratracheal administration. One day before bleomycin instillation, mice were started on treatment with $50 \mu \mathrm{g}$ of recombinant IL-7 or vehicle control followed by administration five times per week for 2 weeks by intraperitoneal injection. Fourteen days following bleomycin exposure, mice were euthanized and both lungs were removed for the determination of hydroxyproline, collagen, TGF- $\beta$, and IFN- $\gamma$ content. Separate experiments assessed whether IL-7-mediated antifibrotic activities are IFN- $\boldsymbol{\gamma}$-independent. Two days before bleomycin instillation, treatment was started with $100 \mu \mathrm{g}$ of anti-IFN- $\gamma$ neutralizing antibody or control antibody five times a week for 2 weeks by intraperitoneal injection to neutralize endogenous and IL-7-induced IFN- $\gamma$. In our previous studies we found that this dose of anti-IFN- $\gamma$ neutralizing antibody had the capacity to neutralize $>95 \%$ circulating IFN- $\gamma$ in vivo (data not shown).

TGF- $\beta$ and IFN- $\gamma$ ELISA. Mouse anti-human TGF- $\beta$ or IFN- $\gamma$ monoclonal capture antibody and biotinylated detecting antibody pairs were obtained from R\&D Systems Inc. Monoclonal anti-TGF- $\beta$ antibody measures TGF- $\beta 1,2$, and 3 . ELISA was performed based on a horseradish peroxidase method as described previously (14-16). OD of each sample after color development was determined with a microplate reader (Dynatech Laboratories, Chantilly, Virginia, USA) at $450 \mathrm{~nm}$.

$R T$-PCR. The human Smad7 sense primer (5'-AGC ACA CCA GCT CGG GGT TGA T- $3^{\prime}$ ) and antisense primer ( $5^{\prime}$ AAC GAT CTG CGC TCG TCC GGC G-3') correspond to the coding regions 308-330 and 872-849 of the human Smad7 cDNA with amplified DNA size of $564 \mathrm{bp}$. The 
housekeeping gene GAPDH was used as control for semiquantification. High-quality total RNA from $10^{7}$ cells each of NF or PFF samples with or without IL-7 treatment $(100 \mathrm{ng} / \mathrm{ml})$ for 24 hours was prepared by the TRIzol method (GIBCO BRL; Life Technologies Inc., Grand Island, New York, USA). RT-PCR was performed at $42^{\circ} \mathrm{C}$ for 1 hour followed by 35 cycles of amplification using a PTC-100 programmable thermal cycler (MJ Research Inc., Watertown, Massachusetts, USA).

Collagen assay. Collagen assay was performed using the Sircol collagen assay method (Accurate Chemical \& Scientific Corp., Westbury, New York, USA). Briefly, 1,000 $\mu \mathrm{l}$ of Sircol dye reagent was added to $100 \mu$ l of test samples or collagen standards and incubated at $25^{\circ} \mathrm{C}$ for 30 minutes. After centrifugation at $5,000 \mathrm{~g}$ for 5 minutes, the supernatants were drained off and discarded. One thousand $\mu \mathrm{l}$ of $0.5 \mathrm{~N} \mathrm{NaOH}$ was added to the collagenbound dye pellet to release the bound dye into solution. The OD of each sample was determined with a microplate reader (Dynatech Laboratories) at $540 \mathrm{~nm}$. Hydroxyproline assay. Lungs were harvested on day 14 after bleomycin administration $(18,19)$. One-half milliliter of lung homogenate was digested in $1 \mathrm{ml}$ of $6 \mathrm{~N}$ $\mathrm{HCl}$ for 8 hours at $120^{\circ} \mathrm{C}$. Five microliters of citrate/acetate buffer ( $5 \%$ citric acid, $7.24 \%$ sodium acetate, $3.4 \%$ sodium hydroxide, and $1.2 \%$ glacial acetic acid, $\mathrm{pH}$ 6.0) and $100 \mu \mathrm{l}$ of chloramines-T solution (282 $\mathrm{mg}$ of chloramines- $\mathrm{T}, 2 \mathrm{ml}$ of $n$-propanol, $2 \mathrm{ml}$ of $\mathrm{H}_{2} \mathrm{O}$, and $16 \mathrm{ml}$ of citrate/acetate buffer, $\mathrm{pH}$ 6.0) were added to $5 \mu \mathrm{l}$ of sample and incubated for 20 minutes. Next, $100 \mu \mathrm{l}$ of Ehrlich's solution (2.5 g of 4-(dimethylamino)benzaldehyde, $9.3 \mathrm{ml}$ of $n$-propanol, $3.9 \mathrm{ml}$ of $70 \%$ perchloric acid) was added to each sample and incubated for 15 minutes at $65^{\circ} \mathrm{C}$. The OD was determined at $550 \mathrm{~nm}$ on a DU 640 spectrophotometer (Beckman Instruments Inc., Fullerton, California, USA). Hydroxyproline (Sigma Chemical Co., St. Louis, Missouri, USA) concentrations from $0-10 \mu \mathrm{g} / \mathrm{ml}$ were used to construct a standard curve.

Statistical analysis. All in vitro results are representative of at least three independent experiments performed in triplicate. In vivo experiments were performed with 10 mice per group, which yields a power of $>90 \%$ to detect any difference among the treatment groups $(\alpha=0.05)$. Significance of differences between experimental and control values was calculated using the Student's $t$ test.

\section{Results}

IL-7 decreases TGF- $\beta$ production by PFFs. Based on our previous studies in murine macrophages (14), murine fibrosarcomas (15), and human tumors (16), we hypothesized that IL-7 also would have the capacity to decrease fibroblast TGF- $\beta$ production. In human primary cultures of pulmonary fibroblasts isolated from patients with IPF (20), we found that IL-7 $(100 \mathrm{ng} / \mathrm{ml})$ has the capacity to downregulate the production of TGF- $\beta$, which was quantified by ELISA using $m A b$ 's recognizing TGF- $\beta 1,2$, and 3 (Figure 1a). IL-7, in contrast, does not alter TGF- $\beta$ production in the primary cul- tures of normal fibroblasts derived from lung resection specimens from patients who do not have IPF (Figure 1b). Both PFFs and NFs express the IL-7 receptor (IL-7R). No difference was detected in the level of PFF and NF IL-7R expression when assessed by flow cytometry (data not shown). Because IFN- $\gamma$ has previously been shown to decrease TGF- $\beta$ production, we compared the capacities of IFN- $\gamma$ and IL-7 to downregulate TGF- $\beta$ and sought to determine whether their effects were additive. Both PFF and NF were tested and were devoid of IFN- $\gamma$ in the culture supernatant (data not shown). We exposed PFF and NF to IL-7, IFN- $\gamma$ (500 units/ml), IL-7 plus IFN- $\gamma$, or IL-7 plus anti-IFN- $\gamma$ monoclonal neutralizing antibody $(5 \mu \mathrm{g} / \mathrm{ml}$ ) (Figure 1, $\mathrm{c}$ and d). Whereas NF responded only to IFN- $\gamma$ by downregulating TGF- $\beta$ production, PFF showed decreased TGF- $\beta$ following exposure to either IL-7 or IFN- $\gamma$. As predicted, the addition of anti-IFN- $\gamma$ antibody had no effect on IL-7-mediated downregulation of TGF- $\beta$ because fibroblasts did not produce IFN- $\gamma$. The results suggest that IL-7 is signaling downregulation of TGF- $\beta$ in an IFN- $\gamma$-independent manner and that PFF may have an accessory IL-7 signaling pathway or a different regulatory mechanism that is not operative in NF.

$I L-7 R$ signaling requires an intact JAK1/STAT1 signaling pathway. Activation of the JAK/STAT pathway has been implicated in IL-7R signaling $(21,22)$. Accordingly, we tested whether the capacity of IL-7 to decrease TGF- $\beta$ production was mediated through JAK/STAT signaling. Using JAK1-deficient (U4A) and STAT1-deficient (U3A) mutant fibroblast cell lines, we observed that IL-7 had no effect on TGF- $\beta$ production (Figure 2). However, when an intact JAK/STAT signaling pathway was reconstituted by complementing U4A cells with JAK1 expression (U4A/JAK1), the fibroblasts responded to IL-7 by downregulating TGF- $\beta$ production in a

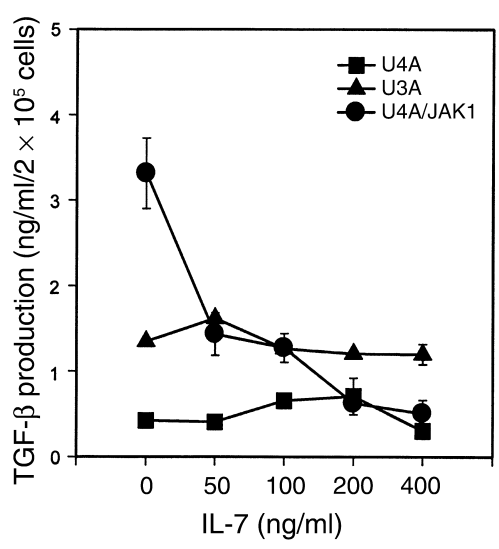

\section{Figure 2}

IL-7R signaling requires an intact JAK1/STAT1 pathway. U4A, U3A, and U4A/JAK1 fibroblast cell lines were cultured in medium containing variable doses of recombinant IL-7 for a 24-hour incubation. Culture supernatants were assayed for TGF- $\beta$ by ELISA. Whereas TGF- $\beta$ production remained unchanged in the U4A and U3A cells, IL-7 decreased TGF- $\beta$ production in a dose-dependent manner in U4A/JAK1 cells $(P<0.01$ at $50-400 \mathrm{ng} / \mathrm{ml} \mathrm{IL-7})$. 


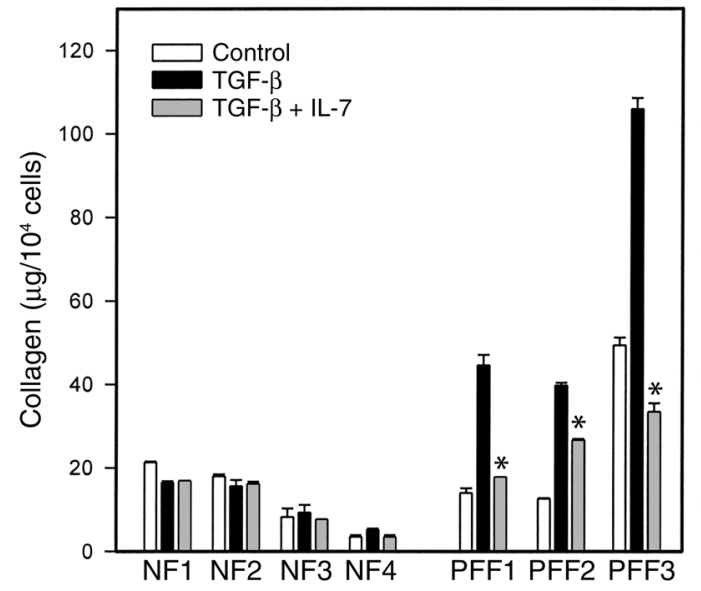

Figure 3

IL-7 inhibits TGF- $\beta$-induced collagen synthesis in PFF. PFF $(n=3)$ and $\mathrm{NF}(n=4)$ were cultured in medium alone, medium containing TGF- $\beta 1$, or the combination of TGF- $\beta 1$ plus IL-7 for 24 hours. The collagen content from the cell lysates was determined. IL-7 inhibits TGF- $\beta$-induced collagen synthesis in primary PFF but not in NF. ${ }^{*} P<0.01$.

dose-dependent manner (Figure 2). These findings indicate that the downregulation of TGF- $\beta$ production in fibroblasts by IL-7 requires an intact JAK1/STAT1 signal transduction pathway. In contrast to fibroblasts from nonfibrotic lungs, the U4A/JAK1 fibroblast cell line shows decreased TGF- $\beta$ in response to IL-7 and, in this regard, is similar to PFFs.

IL-7 decreases TGF- $\beta$-induced collagen synthesis. In addition to decreasing TGF- $\beta$ production, another important contribution for a cytokine used therapeutically to modulate the fibrotic response would be the capacity to inhibit the effects of TGF- $\beta$ signaling $(21,22)$. TGF- $\beta$ is known to stimulate both fibroblast proliferation (23, 24) and collagen deposition (25). To test whether IL-7 could modulate TGF- $\beta$-induced collagen synthesis, we exposed PFF and NF to activated recombinant TGF- $\beta 1$ alone $(10 \mathrm{ng} / \mathrm{ml})$ or TGF- $\beta$ plus IL-7 $(100 \mathrm{ng} / \mathrm{ml})$. When used at this concentration, TGF- $\beta$ did not significantly impact fibroblast proliferation at 24 hours in either NF or PFF (data not shown). When stimulated with TGF- $\beta$, PFF but not NF showed enhanced collagen production (Figure 3). Following preincubation in IL-7 (100 ng/ml) for 2 hours before the addition of TGF- $\beta 1$, there was a marked reduction in the capacity for TGF- $\beta$ to elicit fibroblast collagen synthesis in PFF (Figure 3). Interestingly, IL-7 had no effect on the constitutive level of collagen synthesis. This suggests that IL-7 inhibits collagen synthesis by blocking TGF- $\beta$ signaling in PFF.

IL-7 inhibits TGF- $\beta$ signaling through the induction of Smad7. TGF- $\beta$ signaling and regulation are mediated by a family of SMAD proteins $(21,22)$. Among the SMAD family, Smad7 is the major inhibitor of TGF- $\beta$ signaling. Previous studies indicate that IFN- $\gamma$ has the capacity to regulate Smad7 through the JAK/STAT pathway (21). Based on our observation that IL-7 also mediates its effects in PFF through the
JAK/STAT pathway, we hypothesized that IL-7 interfered with TGF- $\beta$ signaling and, therefore, decreased TGF- $\beta$-induced collagen synthesis by enhancing Smad7 expression in PFF. As shown in Figure 4a, IL-7 increased Smad7 in pulmonary fibroblasts. In order to determine whether the IL-7-induced increase in Smad7 expression was the cause of IL-7-mediated inhibition of collagen synthesis, PFF were transduced with a Smad7 dominant negative construct. The levels of collagen synthesis in response to IL-7 were evaluated in both untransduced fibroblasts and the Smad7 dominant negative-transduced (Smad7DNtransduced) cells. In the presence of IL-7, Smad7DNtransduced fibroblasts maintained high-level TGF$\beta$-induced collagen synthesis, indicating that an IL-7-mediated increase in Smad7 led to inhibition of TGF- $\beta$ signaling (Figure $4 b$ ).

IL-7 in hibits bleomycin-induced pulmonary fibrosis in vivo. Based on the capacity of IL-7 to downregulate TGF- $\beta$ production and block TGF- $\beta$ signaling in human PFF, we tested whether recombinant IL-7 would limit the

a
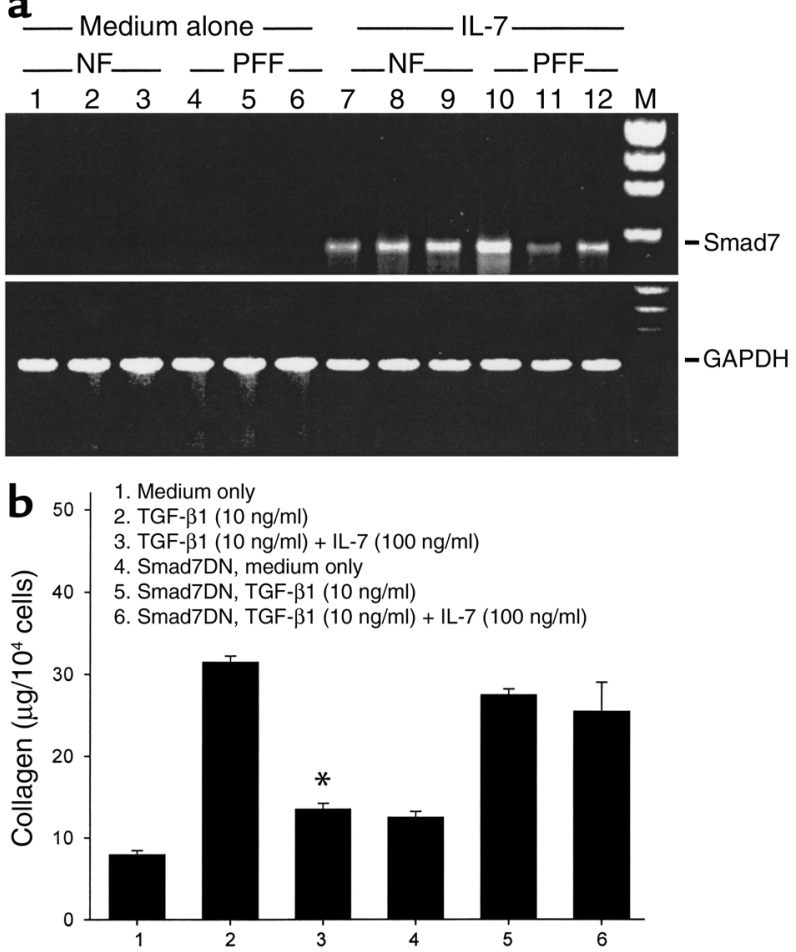

Figure 4

IL-7 inhibits TGF- $\beta$ signaling through the induction of Smad7. (a) The Smad7 expression from PFF $(n=3)$ or NF $(n=3)$ was determined by RT-PCR. The upper panel shows amplified Smad7 PCR product; the lower panel shows GAPDH expression in the same samples. Lanes 1-3 and 7-9 are NF. Lanes 4-6 and 10-12 are PFF. Lanes 1-6: Fibroblasts were cultured in medium alone. Lanes 7-12: Fibroblasts were incubated in IL-7 for 24 hours. M, molecular weight marker. IL-7 increases Smad7 mRNA expression in both NF and PFF. (b) PFF and Smad7DN-transduced PFF were incubated in medium alone, medium containing TGF- $\beta 1$, or medium containing TGF- $\beta 1$ plus IL-7 for a 24-hour incubation. IL-7-mediated inhibition of collagen synthesis by TGF- $\beta$ was not observed in Smad7DN-transduced PFF. ${ }^{*} P<0.01$. 

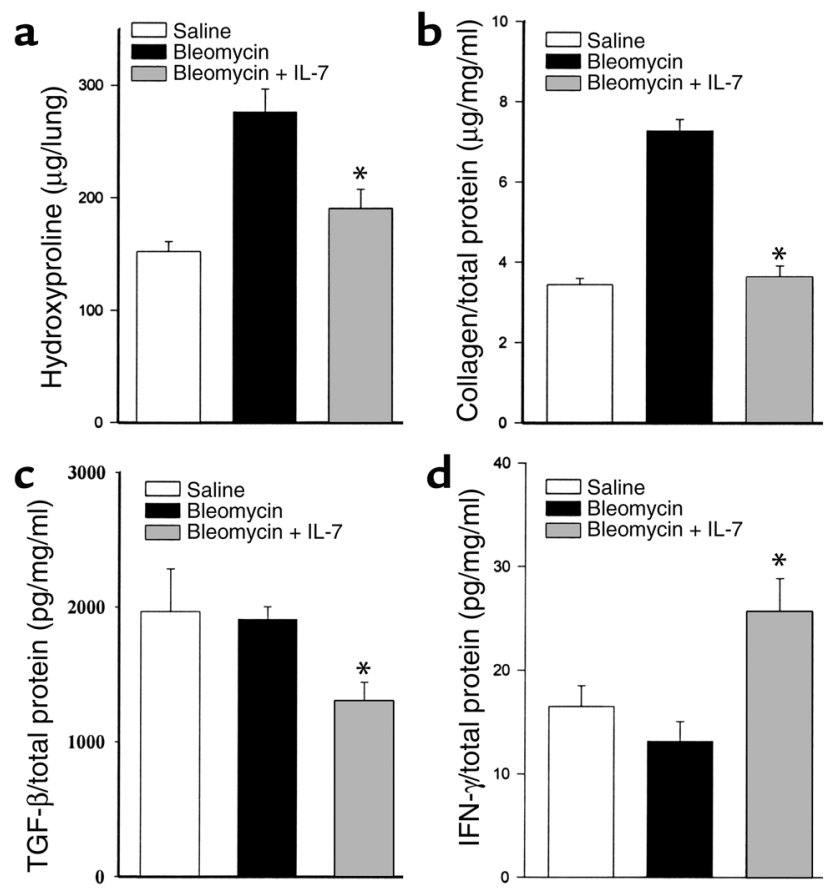

\section{Figure 5}

IL-7 inhibits bleomycin-induced pulmonary fibrosis in vivo. Lung homogenates from each group of mice $(n=10)$ were assayed for hydroxyproline (a), collagen (b), TGF- $\beta$ (c), and IFN- $\gamma(\mathbf{d})$ content. The data represent the average values of each group of mice with standard deviation. IL-7 decreases hydroxyproline and collagen levels, decreases TGF- $\beta$, and increases IFN- $\gamma$ in bleomycin-treated lung homogenates. ${ }^{*} P<0.01 \mathrm{IL}-7+$ bleomycin compared with bleomycin alone.

development of bleomycin-induced pulmonary fibrosis in vivo. Consistent with our in vitro findings, recombinant IL-7 administration ( $5 \mu \mathrm{g} /$ mouse five times per week for 2 weeks) by intraperitoneal injection decreased bleomycin-induced pulmonary fibrosis, as indicated by a decrease in hydroxyproline and collagen content in mouse lung homogenates (Figure 5, a and b). IL-7 administration also decreased TGF- $\beta$ production but increased IFN- $\gamma$ secretion in lung homogenates from mice with bleomycin-induced pulmonary fibrosis (Figure $5, \mathrm{c}$ and d). These results indicated that IL-7 has in vivo antifibrotic activities. Because IL-7-treated lungs in the bleomycin-induced pulmonary fibrosis murine models had higher levels of IFN- $\gamma$, we speculated that the IL-7-mediated antifibrotic response may depend, in part, on the overproduction of IFN- $\gamma$. In preliminary studies, we have determined that $1 \mu \mathrm{g}$ of the purified anti-mouse IFN- $\gamma$ monoclonal neutralizing antibody used in these studies was able to neutralize 400-800 ng of mouse IFN- $\gamma$ in vitro (data not shown). Administration of anti-IFN- $\gamma$ neutralizing antibody intraperitoneally at a concentration of $100 \mu \mathrm{g}$ per injection per mouse five times a week for 2 weeks had the capacity to neutralize more than $95 \%$ of circulating IFN- $\gamma$ in vivo (data not shown). To determine the importance of IFN- $\gamma$ in the IL-7-mediated antifibrotic activities in vivo, we repeated the in vivo experiment (Figure 5) using anti-IFN- $\gamma$ neutralizing antibody $(100 \mu \mathrm{g} /$ mouse five times per week for 2 weeks) administered by intraperitoneal injection and assessed hydroxyproline and collagen levels. The results indicated that IL-7 decreased bleomycin-induced pulmonary fibrosis in an IFN- $\gamma$-independent manner (Figure 6).

\section{Discussion}

The TGF- $\beta$ superfamily is a group of multifunctional regulatory peptides that have a broad array of activities in cell growth and differentiation (23-34). TGF- $\beta$ plays a pivotal role in tissue fibrosis (23-34), including pulmonary fibrosis $(23-26,31-34)$. Within the normal lung, TGF- $\beta 1$ is the most abundant isoform and is mostly expressed in interstitial fibroblasts and bronchiolar epithelial cells $(24,25,31-33)$. Within lungs developing pulmonary fibrotic responses, TGF- $\beta 1$ is found to be overexpressed in a broad range of cells including fibroblasts, macrophages, and epithelial and endothelial cells (23-34). The pathogenesis of pulmonary fibrosis includes the deterioration of the normal homeostatic mechanisms regulating the equilibrium between the synthesis and breakdown of the ECM (24). Overexpression of TGF- $\beta$ contributes to the dysregulation of the normal homeostasis at multiple levels: it enhances synthesis and deposition of ECM components including collagen and can also alter the balance of matrix metalloproteinases and their inhibitors $(24,25,30-34)$. Based on the importance of TGF- $\beta$ in the pathogenesis of pulmonary fibrosis, in the current study IL-7 was chosen for evaluation, because we have previously found that IL-7 downregulates macrophage (14), fibrosarcoma, and melanoma $(15,16)$ production of TGF- $\beta$.

The current findings indicate that recombinant IL-7 significantly decreases TGF- $\beta$ production in PFF but
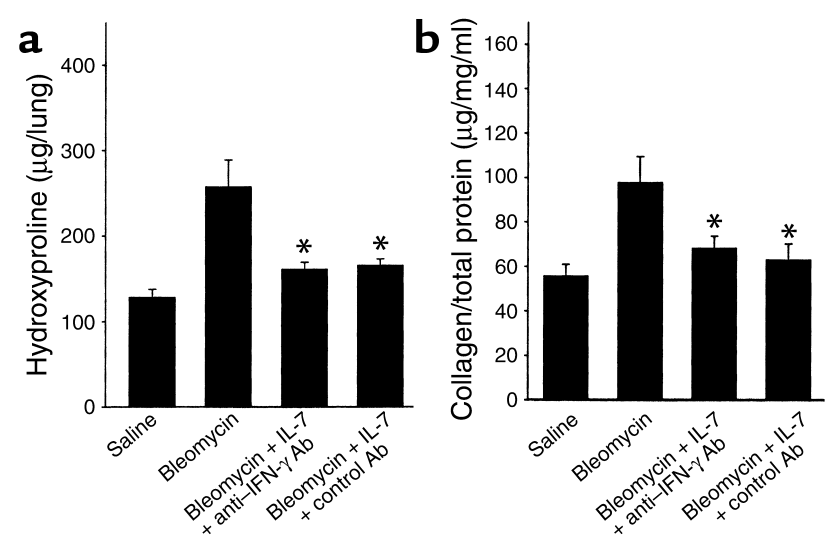

\section{Figure 6}

IL-7-mediated antifibrotic activities are IFN- $\gamma$-independent. Lung homogenates from each group of mice $(n=10)$ were assayed for hydroxyproline (a) and collagen (b). The data represent the average values of each group of mice with standard deviation. IL-7 decreases hydroxyproline and collagen levels independent of IFN- $\gamma$ in bleomycin-treated lung homogenates. ${ }^{*} P<0.01 \mathrm{IL}-7+$ bleomycin compared with bleomycin alone. 
not in NF. Because IL-7R expression did not differ between PFF and NF cells, we speculate that downstream regulatory signaling events account for this difference in IL-7 responsiveness. Further studies will be necessary to define these differences. As is documented for several other cytokines, IL-7 has previously been found to signal by the JAK1/STAT1-dependent pathway in lymphocytes (35). Based on these previous studies, we tested whether IL-7 could mediate a decrease in fibroblast TGF- $\beta$ in the absence of JAK1/STAT1. Our current findings document, for the first time to our knowledge, that JAK1/STAT1-dependent signaling is operative for IL-7 in human fibroblasts.

JAK/STAT-dependent signaling in fibroblasts has been previously found to be critical for the capacity of IFN- $\gamma$ to upregulate Smad7 and inhibit TGF- $\beta$ signaling (21). IFN- $\gamma$ signaling rapidly increases the expression of Smad7, causing the inhibition of Smad3 phosphorylation and subsequently loss of TGF- $\beta$ signaling to the nucleus $(21,22)$. These findings indicate a mechanism of transmodulation between the STAT and SMAD signal transduction pathways. In our current studies, in addition to the capacity to downregulate TGF- $\beta$ production, IL-7 was found to potently induce Smad7 and subsequently block TGF- $\beta$ signaling. The functional manifestation of this blockade in TGF- $\beta$ signaling is demonstrated by a profound decrease in the TGF- $\beta$-induced collagen synthesis in PFF. IFN- $\gamma$ is known to decrease both TGF- $\beta$ production and signaling and has shown promise in preliminary clinical trials (4). Although IL-7 is known to induce IFN- $\gamma$, our current study indicates that IL-7 mediates potent antifibrotic responses both in vitro and in vivo in an IFN- $\gamma$-independent manner.

A variety of models have been used to assess potential therapeutic interventions for pulmonary fibrosis $(18-20,32,33,36-46)$. In this study we used the bleomycin model because of its widespread use for this purpose (18-20, 32, 33, 36-39, 41-46). However, bleomycin has been known to induce acute lung injury, thus initiating the process of bleomycin-induced fibrosis (39). Although the bleomycin model does not fully replicate the human disease that is clinically recognized as IPF, we chose to use this model because bleomycin induces a TGF- $\beta$-dependent induction of pulmonary fibrosis that is accompanied by increased collagen and hydroxyproline synthesis $(18-20,32,33,37,41,42$, 44-46). For example, studies indicate that administration of anti-TGF- $\beta$ antibodies (32), soluble TGF- $\beta$ receptors (41), the TGF- $\beta$ inhibitor decorin $(43,44)$, or Smad7 gene transfer (37) limits bleomycin-induced pulmonary fibrosis, thus strongly implicating a pathogenic role for TGF- $\beta$ in this model. Consistent with our in vitro findings in human fibroblasts derived from pulmonary fibrosis biopsies, IL-7 inhibited pulmonary fibrosis in vivo in the bleomycin model.

Although investigators have shown that TGF- $\beta$ can impact the phenotype and function of normal fibroblasts, studies also demonstrate marked differences between normal and fibrosis fibroblasts (26-30). The differences in TGF- $\beta$-induced collagen synthesis by NF and PFF may be due to a differential response of these cell populations to this cytokine and are consistent with previously reported observations (26-30). In our current study, recombinant TGF- $\beta$ did not affect collagen synthesis in normal fibroblasts under the conditions and concentrations evaluated. Because activated recombinant TGF- $\beta 1$ was used in these studies, potential differences in TGF- $\beta$ activation state could not account for these observations. Our findings are consistent with previous investigations that revealed marked differences in TGF- $\beta$-dependent responses in fibroblasts from different developmental or pathogenic states (27-30). The precise mechanism of the differential response to TGF- $\beta$ by PFF and NF remains unclear and requires further investigation.

The current study was designed to assess the effectiveness of IL-7 as a potential antifibrotic agent and to begin to understand the capacity of this cytokine to regulate TGF- $\beta$ production and signaling. The full clinical relevance of these findings will require further investigation, including an assessment of the time course of IL-7 efficacy following bleomycin exposure. Novel treatment paradigms are being developed based on a more complete understanding of the pathogenesis of IPF (1-4). In marked contrast to the current forms of therapy for IPF that use steroids and immunosuppressive agents, IL-7 potently enhances cell-mediated immunity. In addition to its previously documented capacity to induce IFN- $\gamma$, we report here that IL-7 downregulates both TGF- $\beta$ production and signaling in pulmonary fibroblasts. Our results suggest that IL-7 is a potentially therapeutic cytokine for IPF.

\section{Acknowledgments}

This work was supported by NIH grants P50 CA-90388 and R01 CA-085686 (to S.M. Dubinett), P01 HL-67665 (to R.M. Strieter and M.P. Keane), R01 CA-78654 (to R.K. Batra), and P01 HL-03906 (to M.P. Keane); the American Lung Association; Medical Research Funds from the Department of Veteran Affairs; the Research Enhancement Award Program; and the Tobacco-Related Disease Research Program of the University of California. We thank George R. Stark for generously providing U3A, U4A, and U4A/JAK1 cell lines, and Rik Derynk for providing the Smad7 retroviral vector.

\footnotetext{
1. Gross, T.J., and Hunninghake, G.W. 2001. Idiopathic pulmonary fibrosis. N. Engl. J. Med. 345:517-525.

2. Sheppard, D. 2001. Pulmonary fibrosis: a cellular overreaction or a failure of communication? J. Clin. Invest. 107:1501-1502.

3. Lynch, J.P., 3rd, and McCune, W.J. 1997. Immunosuppressive and cytotoxic pharmacotherapy for pulmonary disorders. Am. J. Respir. Crit. Care Med. 155:395-420.

4. DuBois, R.M. 1999. Interferon gamma-1 $\beta$ for the treatment of idiopathic pulmonary fibrosis. N. Engl. J. Med. 341:1302-1304.

5. Hunt, P., et al. 1987. A single bone marrow-derived stromal cell type supports the in vitro growth of early lymphoid and myeloid cells. Cell. 48:997-1007.

6. Namen, A.E., et al. 1988. Stimulation of B-cell progenitors by cloned murine interleukin-7. Nature. 333:571-573.

7. Conlon, P.J., et al. 1989. Murine thymocytes proliferate in direct response to interleukin-7. Blood. 74:1368-1373.
} 
8. Watson, J.D., Morrissey, P.J., Namen, A.E., Conlon, P.J., and Widmer, M.B. 1989. Effect of IL-7 on the growth of fetal thymocytes in culture. J. Immunol. 143:1215-1222.

9. Miller, P.W., et al. 2000. Intratumoral administration of adenoviral interleukin 7 gene-modified dendritic cells augments specific antitumor immunity and achieves tumor eradication. Hum. Gene Ther. 11:53-65.

10. Borger, P., Kauffman, H., Postma, D., and Vellenga, E. 1996. IL-7 differentially modulates the expression of IFN- $\gamma$ and IL- 4 in activated human $\mathrm{T}$ lymphocytes by transcriptional and post-transcriptional mechanisms. J. Immunol. 156:1333-1338.

11. Mehrotra, P.T., Grant, A.J., and Siegel, J.P. 1995. Synergistic effects of IL-7 and IL-12 on human T cell activation. J. Immunol. 154:5093-5102.

12. Armitage, R.J., Macduff, B.M., Ziegler, S.F., and Grabstein, K.H. 1992. Multiple cytokine secretion by IL-7-stimulated human T cells. Cytokine. 4:461-469.

13. Armitage, R.J., Macduff, B.M., Ziegler, S.F., and Grabstein, K.H. 1990. Regulation of human $\mathrm{T}$ cell proliferation by IL-7. J. Immunol. 144:938-941.

14. Dubinett, S.M., Huang, M., Dhanani, S., Wang, J., and Beroiza, T. 1993. Down-regulation of macrophage transforming growth factor- $\beta$ messenger RNA expression by interleukin-7. J. Immunol. 151:6670-6680.

15. Dubinett, S., et al. 1995. Down-regulation of murine fibrosarcoma transforming growth factor- $\beta 1$ expression by interleukin 7. J. Natl. Cancer Inst. 87:593-597.

16. Miller, A.R., et al. 1993. Transduction of human melanoma cell lines with the human interleukin-7 gene using retroviral-mediated gene transfer: comparison of immunological properties with IL-2. Blood. 82:3686-3694.

17. Spitalny, G.L. 1984. Neutralization of the antiviral activity of 10 units of murine interferon- $\gamma$ requires $30 \mathrm{ng}$ of R4-6A2 antibody. J. Exp. Med. 159:1560-1565.

18. Keane, M.P., et al. 1999. IFN-gamma-inducible protein-10 attenuates bleomycin-induced pulmonary fibrosis via inhibition of angiogenesis. J. Immunol. 163:5686-5692.

19. Keane, M.P., et al. 1999. Neutralization of the CXC chemokine, macrophage inflammatory protein-2, attenuates bleomycin-induced pulmonary fibrosis. J. Immunol. 162:5511-5518.

20. Keerthisingam, C.B., et al. 2001. Cyclooxygenase-2 deficiency results in a loss of the anti-proliferative response to transforming growth factorbeta in human fibrotic lung fibroblasts and promotes bleomycininduced pulmonary fibrosis in mice. Am. J. Pathol. 158:1411-1422.

21. Ulloa, L., Doody, J., and Massague, J. 1999. Inhibition of transforming growth factor-beta/SMAD signalling by the interferon-gamma/STAT pathway. Nature. 397:710-713.

22. Massague, J. 1998. TGF- $\beta$ signal transduction. Annu. Rev. Biochem. 67:753-791.

23. Border, W.A., and Noble, N.A. 1994. Transforming growth factor beta in tissue fibrosis. N. Engl. J. Med. 331:1286-1292.

24. Broekelmann, T.J., Limper, A.H., Colby, T.V., and McDonald, J.A. 1991. Transforming growth factor beta 1 is present at sites of extracellular matrix gene expression in human pulmonary fibrosis. Proc. Natl. Acad. Sci. USA. 88:6642-6646.

25. Khalil, N., Bereznay, O., Sporn, M., and Greenberg, A.H. 1989. Macrophage production of transforming growth factor beta and fibroblast collagen synthesis in chronic pulmonary inflammation. $J$. Exp. Med. 170:727-737.

26. Ramos, C., et al. 2001. Fibroblasts from idiopathic pulmonary fibrosis and normal lungs differ in growth rate, apoptosis, and tissue inhibitor of metalloproteinases expression. Am. J. Respir. Cell Mol. Biol. 24:591-598.

27. Reisdorf, P., Lawrence, D.A., Sivan, V., Klising, E., and Martin, M.T.
2001. Alteration of transforming growth factor-beta1 response involves down-regulation of Smad3 signaling in myofibroblasts from skin fibrosis. Am. J. Pathol. 159:226-272.

28. Chin, G.S., et al. 2001. Differential expression of transforming growth factor-beta receptors I and II and activation of Smad 3 in keloid fibroblasts. Plast. Reconstr. Surg. 108:423-429.

29. Kishi, K., Nakajima, H., and Tajima, S. 1999. Differential responses of collagen and glycosaminoglycan syntheses and cell proliferation to exogenous transforming growth factor beta 1 in the developing mouse skin fibroblasts in culture. Br. J. Plast. Surg. 52:579-582.

30. Hakenjos, L., Bamberg, M., and Rodemann, H.P. 2000. TGF-beta1mediated alterations of rat lung fibroblast differentiation resulting in the radiation-induced fibrotic phenotype. Int. J. Radiat. Biol. 76:503-509.

31. Westergren-Thorsson, G., et al. 1993. Altered expression of small proteoglycans, collagen, and transforming growth factor-beta 1 in developing bleomycin-induced pulmonary fibrosis in rats. J. Clin. Invest. 92:632-637.

32. Giri, S.N., Hyde, D.M., and Hollinger, M.A. 1993. Effect of antibody to transforming growth factor beta on bleomycin induced accumulation of lung collagen in mice. Thorax. 48:959-966.

33. Zhang, K., Flanders, K.C., and Phan, S.H. 1995. Cellular localization of transforming growth factor-beta expression in bleomycin-induced pulmonary fibrosis. Am. J. Pathol. 147:352-361.

34. Coker, R.K., et al. 1996. Diverse cellular TGF-beta 1 and TGF-beta 3 gene expression in normal human and murine lung. Eur. Respir. J. 9:2501-2507.

35. van der Plas, D.C., et al. 1996. Interleukin-7 signaling in human B cell precursor acute lymphoblastic leukemia cells and murine BAF3 cells involves activation of STAT1 and STAT5 mediated via the interleukin7 receptor alpha chain. Lenkemia. 10:1317-1325.

36. Bowden, D.H. 1984. Unraveling pulmonary fibrosis: the bleomycin model. Lab. Invest. 50:487-488.

37. Nakao, A., et al. 1999. Transient gene transfer and expression of Smad7 prevents bleomycin-induced lung fibrosis in mice. J. Clin. Invest. 104:5-11.

38. Krishna, G., et al. 2001. PG490-88, a derivative of triptolide, blocks bleomycin-induced lung fibrosis. Am. J. Pathol. 158:997-1004.

39. Hoyt, D.G., and Lazo, J.S. 1992. Murine strain differences in acute lung injury and activation of poly(ADP-ribose) polymerase by in vitro exposure of lung slices to bleomycin. Am. J. Respir. Cell Mol. Biol. 7:645-651.

40. Christensen, P.J., Goodman, R.E., Pastoriza, L., Moore, B., and Toews, G.B. 1999. Induction of lung fibrosis in the mouse by intratracheal instillation of fluorescein isothiocyanate is not T-cell-dependent. Am. J. Pathol. 155:1773-1779.

41. Wang, Q., et al. 1999. Reduction of bleomycin induced lung fibrosis by transforming growth factor beta soluble receptor in hamsters. Thorax. 54:805-812.

42. Corbel, M., et al. 2001. Inhibition of bleomycin-induced pulmonary fibrosis in mice by the matrix metalloproteinase inhibitor batimastat. J. Pathol. 193:538-545.

43. Giri, S.N., et al. 1997. Antifibrotic effect of decorin in a bleomycin hamster model of lung fibrosis. Biochem. Pharmacol. 54:1205-1216.

44. Kolb, M., et al. 2001. Transient transgene expression of decorin in the lung reduces the fibrotic response to bleomycin. Am. J. Respir. Crit. Care Med. 163:770-777.

45. Gurujeyalakshmi, G., and Giri, S.N. 1995. Molecular mechanisms of antifibrotic effect of interferon gamma in bleomycin-mouse model of lung fibrosis: downregulation of TGF-beta and procollagen I and III gene expression. Exp. Lung Res. 21:791-808.

46. Cooper, J.A., Jr. 2000. Pulmonary fibrosis: pathways are slowly coming into light. Am. J. Respir. Cell Mol. Biol. 22:520-523. 